vol. $27-n^{\circ} 2$ | 2011

Numéro ouvert

\title{
La négociation des frontières ethniques dans l'espace scolaire : un regard québécois
}

The Negotiation of Ethnic Relations in School Space: A Quebec's Eye View

La negociación de fronteras étnicas en el espacio escolar: una mirada

quebequesa

\section{Stéphanie Tremblay}

\section{OpenEdition}

Journals

\section{Édition électronique}

URL : https://journals.openedition.org/remi/5451

DOI : $10.4000 /$ remi.5451

ISSN : $1777-5418$

\section{Éditeur}

Université de Poitiers

\section{Édition imprimée}

Date de publication : 1 octobre 2011

Pagination : 117-138

ISBN : 979-10-90426-01-6

ISSN : 0765-0752

\section{Référence électronique}

Stéphanie Tremblay, «La négociation des frontières ethniques dans l'espace scolaire : un regard québécois », Revue européenne des migrations internationales [En ligne], vol. 27 - n² | 2011, mis en ligne le 01 octobre 2014, consulté le 14 avril 2022. URL : http://journals.openedition.org/remi/5451 ; DOI : https://doi.org/10.4000/remi.5451 


\title{
La négociation des frontières ethniques dans l'espace scolaire : un regard québécois
}

\author{
Stéphanie TREMBLAY ${ }^{1}$
}

\section{INTRODUCTION}

\begin{abstract}
A u sein des démocraties modernes, l'école joue un rôle crucial dans le maintien ou la transformation des frontières ethniques, puisqu'il s'agit de la principale institution, après la famille, qui prend en charge l'éducation de l'enfant. Mais contrairement au milieu confortable de la famille, l'école doit en quelque sorte créer un environnement " non sécurisé » (Leroux, 2010) de manière à transformer l'enfant en citoyen, par le biais de ses diverses fonctions d'instruction, de socialisation et de qualification. Après avoir examiné la notion conceptuelle de frontière ethnique et illustré ses applications politiques selon divers modèles normatifs de citoyenneté, nous verrons à quel point la prise en compte du pluralisme et des identités communautaires dans l'espace scolaire ne fait pas l'unanimité. En abordant à tour de rôle les grandes fonctions de la scolarisation à travers des exemples tirés entre autres de l'actualité québécoise, nous tenterons néanmoins de dégager quelques balises pour délimiter l'espace acceptable de la diversité culturelle et religieuse en milieu scolaire, en particulier en contexte libéral. En ce qui a trait à la mission d'instruction, nous explorerons notamment cette problématique au prisme des rapports entre religions et science à l'école et de l'équilibre que doit trouver l'État entre une « dictature » gouvernementale et le relativisme absolu des savoirs scolaires. Cette réflexion se prolongera dans une analyse de la mission de socialisation de l'école libérale et du traitement qui devrait être réservé aux écoles ethnoreligieuses au regard des exigences de la citoyenneté. Concernant la fonction de qualification, nous examinerons à quel point la variable « ethnique » continue ou non d'expliquer les échecs scolaires massifs de certains groupes sociaux (notamment au Québec). Cette question nous conduira enfin à nous interroger sur l'offre de mesures compensatoires auprès de
\end{abstract}

1 Doctorante en sciences de l'éducation, Université de Montréal, Centre d'études ethniques des universités montréalaises (CEETUM), CP 6128, Succ. Centre-ville Montréal (Québec) Canada, H3C 3J7 ; stephanie.tremblay.5@umontreal.ca 
différentes communautés, selon qu'elles soient issues de l'immigration ou de minorités nationales.

\section{LA CONSTRUCTION DES FRONTIÈRES ETHNIQUES ET LE RÔLE DE L'ÉDUCATION : QUELQUES APPROCHES THÉO- RIQUES ET LEURS APPLICATIONS POLITIQUES}

Pour réfléchir au rôle de l'éducation dans la construction des frontières ethniques, un examen préalable de la nature et des différentes conceptions de l'ethnicité en vigueur s'impose. Plusieurs débats agitent depuis de nombreuses années le champ de l'ethnicité, mais une constante demeure : le concept d'ethnicité n'est toujours pas éculé, comme le prédisaient pourtant les analystes fonctionnalistes et marxistes, qui anticipaient son effacement au fil du processus de globalisation ou de l'avènement d'une société sans classe (Hervieu-Léger, 1999 : 13-16 ; Juteau, 1999 : 12-13 ; Mc Andrew, 2000 : 4-5). Les théories sociologiques de l'ethnicité et des relations ethniques ont en effet dû adapter leur dispositif d'analyse pour tenir compte non seulement de la persistance des identités ethniques, mais aussi de leur renouvellement multiforme dans la foulée de l' « ethnic revival » des années 1970. Plusieurs sociétés occidentales sont alors devenues le lieu d'expression de revendications ethniques de toutes sortes (Taylor, 1992), de mouvements civiques (comme les Noirs aux États-Unis), régionalistes (tels les Occitans et les Bretons en France), ou encore d'autonomisation (comme les Autochtones au Canada).

Si certains ont décelé dans cette recrudescence des identités ethniques, la confirmation du caractère " essentialiste » ou figé de l'ethnicité (Novak, 1971), d'autres ont interprété cette «nouvelle ethnicité » comme un choix d'association stratégique de certains groupes en vue d'obtenir plus de ressources de l'État (Glazer et Moynihan, 1976). Ce constat a d'ailleurs été conforté par plusieurs recherches en psychologie sociale, qui mettaient en évidence les concepts d' «identités multiples » et de saillance identitaire reflétant l'aspect stratégique de la présentation de l'une ou l'autre de ses faces selon le contexte (Taboada-Leonetti, 1990). De même, la réflexion en sociologie et en anthropologie faisait valoir le déplacement dans le temps des marqueurs culturels caractérisant certaines communautés et permettant de tracer une frontière les séparant des autres (Barth, 1969 ; Mc Andrew, 2000 : 6). Remise en cause par ces divers courants critiques, la perspective essentialiste de l'ethnicité a donc graduellement cédé la place au paradigme constructiviste, qui focalisait sur la « construction » des frontières ethniques, par les communautés elles-mêmes (frontière interne) ou par l'entremise des rapports de pouvoir entre les groupes (frontière externe) (Juteau, 1999).

Même si un certain consensus s'est formé autour de la perspective constructiviste dans l'analyse de l'ethnicité, il n'en demeure pas moins que les auteurs ne s'entendent pas sur la facette à privilégier dans sa définition. Or, loin d'être neutres, ces choix révèlent souvent des positions normatives différentes et ne se prolongent pas dans les mêmes agendas scolaires.

Suivant Juteau (1999) et Mc Andrew (2000), il est possible de distinguer deux grandes perspectives différentes issues du constructivisme. L'une met l'accent sur la 
frontière « externe » de l'ethnicité, c'est-à-dire sur l'influence exercée par le regard du groupe dominant sur la formation de l'identité culturelle d'un groupe, que ce soit dans un contexte de colonisation ou de migration. Dans cette vision de l'ethnicité, l'appartenance ethnique est surtout perçue comme une chimère camouflant des rapports de domination sociale, politique et économique d'un groupe sur les autres. D'orientation marxiste, elle se prolonge aussi dans un modèle politique républicain, qui voit l'École comme un « sanctuaire » aseptisé des diverses tensions de la société civile (Milot et Estivalèzes, 2008). C'est pourquoi en entrant dans l'école républicaine, les futurs citoyens doivent laisser derrière eux leurs particularismes culturels (ethniques, religieux, linguistiques, etc.) qui risqueraient de compromettre l'idéal égalitaire.

Bien qu'elle soit attentive à toutes les fonctions de l'école, cette approche théorique de l'ethnicité valorise en particulier le mandat de transformation des frontières ethniques dans une perspective d'égalisation des conditions individuelles. Dans son versant marxiste, elle s'accompagne généralement d'une pédagogie antiraciste, qui préconise une lecture critique des rapports historiques de domination entre les groupes et des aspects systémiques de la discrimination et du racisme (Potvin et Carr, 2008 ; Dei, 1994). Ce souci est toutefois édulcoré dans la perspective républicaine, qui insiste moins sur la lutte contre les inégalités économiques que sur la sublimation des intérêts individuels dans un projet politique commun, au moyen d'une " éducation civique traditionnelle " (Mc Andrew, 2000 : 14). Mais, comme l'observe Mc Andrew (2000 : 8-9), l'effet pervers de l'ethnicité définie en priorité par sa frontière externe revient souvent à occulter la mémoire et l'identité distincte des groupes ethniques en tant que tels. En affirmant la suprématie de l'égalité économique (marxiste) ou de l'égalité des citoyens (républicaine), autrement dit de la collectivité sur l'individu, cette approche de l'ethnicité peut donc être taxée d'assimilatrice.

L'autre approche, inspirée de l'interactionnisme symbolique, centre plutôt son attention sur l'individu qui fabriquerait lui-même, au contact de ses « autruis significatifs » (famille, communauté, école, etc.), son identité ethnique, en puisant à différentes sources de son environnement social. La synthèse de l'intériorisation passive des rôles transmis par les agents de socialisation (Moi) et de la créativité personnelle de l'individu (Je) génèrerait ainsi un « Soi » (et une identité ethnique) original (Derocher, 2008 : 35-36 ; Mead, 1963). Bien que la vision interactionniste reconnaisse le rôle de l'Autre dans la formation de l'identité ethnique, elle limite surtout cette prise en compte à l'environnement social immédiat de l'individu. Cette approche tend donc à négliger l'impact des barrières systémiques et des rapports de pouvoir intervenant dans la construction des frontières ethniques (Juteau, 1999 : 177-184 ; Mc Andrew, 2000 : 11-12), en particulier dans sa version postmoderne, qui aborde l'individu comme un consommateur d'identités éclectiques sans ancrage " primaire ». Sur le plan politique, "l'ethnicité comme prérogative de l'individu » rejoint essentiellement les fondements normatifs de l'approche politique libérale, telle qu'on la retrouve au Québec et dans plusieurs autres démocraties modernes. Ainsi, cette conception individualiste de l'ethnicité vise à protéger avant tout «l'indépendance morale des individus », selon l'expression de Bourgeault et al. (1995: 85) :

"Plutôt que d'en appeler à la responsabilité de l'État à l'égard du maintien des communautés ou des cultures elles-mêmes, nous préférons soutenir que celui-ci doit assurer, plus modestement, la protection de l'indépendance morale des individus, en considérant qu'ils appartiennent à des groupes de traditions diverses ». 
La reconnaissance de cette indépendance morale est intimement liée à la protection de la liberté de conscience des individus d'adhérer aux convictions de leur choix. L'ouverture à la diversité culturelle et religieuse devient donc généralement un corollaire de la politique libérale puisque les « conceptions de la vie bonne " des individus sont tributaires, dans une certaine mesure, de leurs ancrages culturels, et de leur survie dans le temps (Kymlicka, 1995). Dans l'espace scolaire, "l'ethnicité comme prérogative de l'individu » se traduit ainsi par l'aménagement d'un environnement propice à la délibération et au libre examen par les enfants de diverses valeurs et normes culturelles. L'importance de ce dialogue démocratique est par exemple clairement soulignée dans les finalités du nouveau cours (non confessionnel) d'éthique et culture religieuse au Québec, implanté dans les écoles primaires et secondaires depuis 2008 : "Cette reconnaissance rend possible l'expression de valeurs et de convictions personnelles. Elle s'actualise dans un dialogue empreint d'écoute et de discernement, qui n'admet ni atteinte à la dignité de la personne ni actions pouvant compromettre le bien commun » (MELS, 2008 : 2).

La responsabilité de l'école repose donc sur un équilibre entre les parents, dont l'héritage culturel doit être pris en compte jusqu'à un certain point, l'État, qui doit veiller à assurer une formation civique commune, et l'intérêt de l'enfant, en particulier son autonomie et son jugement critique (Groupe de travail sur la place de la religion à l'école, 1999 : 83-93). Ici, il n'appartient donc ni à l'école de maintenir ou de transformer les frontières ethniques, mais plutôt de laisser les élèves «négocier » eux-mêmes leur identité en dialoguant avec les autres. Il va de soi que ces derniers ne sont pas tous aussi « libres » de choisir cette identité parce qu'ils ne s'inscrivent pas dans la même case de l'échiquier social, ce qui fait dire à plusieurs critiques antiracistes que cette approche de l'éducation présente un côté " fleur bleue ». En effet, cette approche de l'ethnicité aborde peu la dimension macrosociologique des rapports sociaux et le rôle de la « race » dans la genèse des inégalités sociales (Dei, 1994 ; Potvin et Carr, 2008).

Certains vont par ailleurs continuer à définir l'ethnicité exclusivement à partir de sa face « interne » en considérant le patrimoine forgé par une communauté, dont l'histoire, la langue et la religion, comme allant de soi, sans porter attention à la redéfinition possible de certains de ces marqueurs dans le temps. Cette conception de l'ethnicité, équivalente au paradigme essentialiste délaissé par de nombreux analystes, est toujours préconisée, entre autres, par les partisans des écoles séparées (ethniques et religieuses), qui souhaitent assurer le maintien des identités culturelles minoritaires à travers l'école (Stolzenberg, 1993 : 611-628). Selon ce point de vue d'inspiration communautarienne, l'éducation scolaire relève surtout voire exclusivement de la responsabilité des parents et doit à ce titre refléter leurs affiliations religieuses ou ethniques particulières. L'État fait donc preuve d'ingérence s'il impose le même curriculum scolaire à tous les élèves, certains allant même jusqu'à l'accuser de véhiculer une idéologie assimilatrice de «pluralisme normatif » ou d' " humanisme séculier », reléguant au second plan l'enracinement culturel (surtout religieux) des élèves (Stolzenberg, 1993 ; Zine, 2008).

Dans sa version radicale, cette vision de l'éducation pousse même certains parents à nier le rôle que doit jouer l'éducation dans la formation de l'autonomie, le développement de l'esprit critique et l'ouverture au pluralisme, puisque ces valeurs civiques iraient à l'encontre de leurs croyances religieuses. Dans de tels cas, y compris dans certains 
groupes sectaires coupés totalement ou partiellement de la société, la socialisation des enfants s'inscrit en tension avec les valeurs dominantes de la société puisqu'elle s'oriente en priorité vers les convictions religieuses du groupe, soit vers une « rationalité en valeur » (Derocher, 2008 ; Weber, 1995). Nous reviendrons plus loin sur les implications d'une telle vision du monde appuyée inconditionnellement sur des valeurs religieuses et les limites que l'éducation scolaire doit lui imposer dans les démocraties modernes, notamment pour éviter les risques d'anomie.

Pour rendre compte de la complexité des différentes facettes de l'ethnicité, Juteau (1999) propose, en s'inspirant de la « communalisation ethnique » de Weber, d'articuler les faces « interne » et « externe » de l'ethnicité. Ainsi, au même titre que la communalisation, définie par une « croyance subjective » des membres d'un groupe en une origine commune, l'ethnicité chez Juteau se construit d'une part dans le rapport à l'histoire et à la culture d'un groupe social. Cette face « interne » amène les membres d'un groupe ethnique à se percevoir comme appartenant à la même lignée et à se définir par le truchement de marqueurs identitaires spécifiques, comme la langue et la religion, même si ces marqueurs peuvent évoluer dans le temps. Quant à la face « externe » de l'ethnicité, elle concerne davantage la relation aux autres groupes sociaux et aux relations de pouvoir qui s'établissent entre eux et contribuent à façonner, de l'extérieur, la dynamique des rapports ethniques (Juteau, 1999 : 21-37).

Cette approche théorique présente l'avantage certain d'aborder l'ethnicité comme un fait social dynamique plutôt que comme une forme figée en ce qu'elle met surtout l'accent sur le processus de formation de l'ethnicité et les relations sociales qui le sous-tendent plutôt que sur la communauté elle-même. Ainsi, elle contourne à la fois le problème du « primordialisme », postulant que l'histoire crée naturellement des groupes ethniques distincts, et celui d'une lecture strictement marxiste, qui ne voit l'ehtnicité que comme l'émanation des rapports de domination en occultant la mémoire collective des différentes communautés. On pourrait toutefois reprocher à Juteau un certain déterminisme dans sa conceptualisation de l'ethnicité, dans la mesure où elle ne laisse que très peu de place à la subjectivité de l'individu dans l'appropriation et la synthèse qu'il fait de l'identité ethnique de son groupe. Pourtant, la plupart des théories récentes de la socialisation insistent sur le rôle clé de l'individu dans la traduction de l'identité qui lui est assignée : «L'enfant reçoit le "stock de connaissances" comme une vérité absolue et objective, mais il l'intériorise en tant que vérité subjective : la seule qui soit bonne ! " (Juteau, 1999 : 39). C'est une telle reformulation de la théorie initiale de Juteau que propose Mc Andrew en intégrant aux faces interne et externe des frontières ethniques proposées par Juteau la variable du «Sujet », qui incarne le rôle de la créativité individuelle. Elle définit ainsi l'ethnicité comme « le triple produit de la frontière externe, interne et de la médiation du Sujet ». Cela dit, bien qu'elle pointe l'importance de tenir compte du rôle de l'individu dans l'élaboration de sa propre identité ethnique, cette définition ne présente pas le poids respectif des diverses facettes entrant en jeu dans l'ethnicité.

Malgré ce flou théorique, lorsqu'elle se traduit dans des préoccupations pédagogiques, cette vision tryptique de l'ethnicité présente l'avantage de tenir compte à la fois du rôle des rapports de pouvoir entre les groupes sociaux et de l'autonomie de l'individu, libre de « bricoler » son identité avec les possibilités dont il dispose. C'est donc dire 
qu'elle légitime l'intégration d'une perspective antiraciste dans les programmes scolaires, permettant de questionner la reproduction des inégalités entre les groupes, mais aussi la liberté des individus de définir leurs propres convictions morales, favorisant ainsi une prise en compte des expressions du pluralisme dans les normes et pratiques de l'école, selon les cas. Autrement dit, quoique difficile à opérationnaliser une fois pour toutes sur le terrain scolaire, cette perspective constitue une « Voie du milieu », pour reprendre une expression bouddhiste, un idéal axiologique et normatif vers lequel tendre dans une société démocratique aux prises avec des droits et des intérêts sociaux en tension.

\section{PLURALISME, LAÏCITÉ ET ÉDUCATION SCOLAIRE DANS LA PERSPECTIVE LIBÉRALE}

Au Québec, comme dans plusieurs démocraties modernes, les orientations politiques concernant à la fois le domaine de l'immigration, de l'intégration sociale et de l'éducation traduisent surtout une volonté de s'inscrire dans une approche libérale de la citoyenneté ouverte à la prise en compte de la diversité. Même si la conception de l'ethnicité privilégiée dans les différents avis gouvernementaux focalise davantage sur l'individu que sur les communautés, on y sent le souci de reconnaître les enracinements culturels étayant les identités individuelles. C'est du moins ce qui se dégage de l'essentiel des documents émanant des divers organismes gouvernementaux depuis une vingtaine d'années, à commencer par l'Énoncé de politique en matière d'immigration et d'intégration, qui définissait le Québec dès 1990, comme « une société d'accueil résolument francophone, démocratique et pluraliste » (MICC, $1990: 4)$.

\section{La laïcité, un cadre d'aménagement des rapports sociaux}

Ces grandes orientations se prolongent d'abord dans un modèle de laïcité de plus en plus explicite dans les discours publics et dont les linéaments datent du XVIIIe siècle au Québec, même si le concept de « laïcité » est longtemps demeuré « dans l'angle mort des représentations collectives » (Milot, $2002: 19-37)^{2}$. Contrairement aux définitions, souvent rattachées au contexte français, qui associent laïcité et éradication du religieux de l'espace public (Kintzler, 1998 ; Pena-Ruiz, 2001), la laïcité de « reconnaissance », définie par Milot (2002 et 2008), puis reprise dans le rapport Bouchard-Taylor $(2007)^{3}$, constitue un concept opératoire repérable dans différentes sociétés.

2 D'après la thèse développée par Milot dans Laïcité dans le Nouveau Monde.

3 Il s'agit du rapport de la Commission de consultation sur les pratiques d'accommodement reliées aux différences culturelles. Cette commission, mise sur pied le 8 février 2007, était chargée « de dresser le portrait des pratiques d'accommodement qui ont cours au Québec, d'analyser les enjeux qui y sont associés en tenant compte des expériences d'autres sociétés, de mener une vaste consultation sur ce sujet et de formuler des recommandations au gouvernement pour que ces pratiques soient conformes aux valeurs de la société québécoise en tant que société pluraliste, démocratique et égalitaire » (Commission de consultation sur les pratiques d'accommodement reliées aux différences culturelles, 2007). Au cours de son déroulement, la Commission a tenu des consultations publiques et des forums nationaux auprès de divers acteurs sociaux et de citoyens dans plus de dix-sept villes disséminées à travers le Québec. 
Selon Milot (2008 : 17-21), la laïcité comprend quatre principes, soit « l'égalité morale des personnes ou la reconnaissance de la valeur morale égale de chacune d'entre elles », « la liberté de conscience et de religion », « la neutralité de l'État à l'égard des religions » et « la séparation de l'Église et de l'État». Alors que les deux premiers principes renvoient aux prémisses normatives qui doivent fonder les aménagements laïques, les deux derniers font état des moyens concrets devant être déployés pour garantir la mise en œuvre des deux finalités. Cette définition associe donc directement la laïcité à une facette inhérente (et évolutive) de tout État démocratique garantissant les droits individuels de ses citoyens.

Même en l'absence de toute « charte » chapeautant d'en haut l'application des principes de la laïcité, nous retrouvons au sein de la société des balises juridiques qui font directement écho aux assises de la laïcité identifiées par Milot (2002 et 2008). Les droits fondamentaux des chartes canadienne et québécoise, dotés d'un statut supralégislatif, dont l'égalité et la liberté de conscience et de religion, jouissent d'abord d'une portée réelle dans les décisions des tribunaux. En outre, une exigence de séparation s'impose entre les Églises et l'État, comme corollaire aux droits à l'égalité et à la liberté de conscience et de religion (Mc Andrew et al., 2009), tant dans les institutions politiques et les hôpitaux que dans les écoles (depuis 2008) (MELS, 2008). Dans l'éventualité où l'égalité se trouverait tout de même brimée sous l'effet d'une règle neutre en apparence, mais qui pénaliserait un individu en particulier (Bosset, 2007 : 10), un mécanisme juridique « d'accommodement raisonnable » a d'ailleurs été prévu pour corriger la situation et accorder un traitement différentiel à la personne lésée, dans les limites du raisonnable. Né dans le contexte juridique en 1985, dans une cause de la Cour suprême (arrêt O'Malley) entourant un cas de discrimination en emploi, le concept d'accommodement raisonnable fait aujourd'hui l'objet d'une vaste jurisprudence et de balises plus claires, entre autres en milieu scolaire (arrêt Multani, 2006) ${ }^{4}$ même si son usage demeure controversé (Milot, 2002 : 135-137). Plusieurs critiquent en effet les risques de ghettoïsation, d'enfermement communautaire, de retour de la religion dans l'espace public par la porte « de derrière » ou encore de différences dans les droits entraînés par les « accommodements » religieux. Pourtant, l'argumentaire du gouvernement légitimant cette pratique relève précisément de la volonté contraire, soit celle d'assurer une meilleure intégration de tous dans les institutions publiques (Milot, 2002 : 127-135).

En contexte scolaire, une discrimination indirecte pourrait par exemple survenir si le menu d'une cafétéria ne prévoyait pas d'alternative à la viande de porc, ce qui brimerait subrepticement l'égalité des élèves juifs ou musulmans mangeant casher ou halal. La jurisprudence précise à cet égard que l'institution interpellée par une demande d'accommodement raisonnable doit faire des efforts significatifs, sérieux et sincères en vue de trouver un accommodement (Bosset, 2007 : 10), en particulier lorsqu'il s'agit d'une école dont les enfants forment une clientèle captive. En revanche, une telle demande franchit la limite du raisonnable lorsqu'elle implique une « contrainte excessive » pour l'institution

4 À cet égard, c'est surtout la notion de « contrainte excessive » qui sert d'étalon pour jauger le caractère raisonnable ou non de l'accommodement proposé. De plusieurs ordres, la contrainte excessive concerne surtout l'atteinte aux droits d'autrui (intégrité physique, droit à la vie et à la santé, atteinte au moral du personnel) et l'entrave au bon fonctionnement de l'entreprise ou de l'institution en cause (coûts financiers ou matériels). 
ou porte atteinte aux droits d'autrui (Commission des droits de la personne et des droits de la jeunesse, 1995). Le cas récent de la jeune étudiante fréquentant un centre de francisation vêtue d'une burqa, qui a été expulsée de son cours puisqu'elle refusait de l'enlever, a contribué à définir une telle frontière ${ }^{5}$. La direction du centre, appuyée par le gouvernement, a en effet déclaré que peu importe le vêtement (religieux ou non), un visage complètement masqué (par exemple sous une burqa) nuisait au fonctionnement de l'institution scolaire et de la poursuite de ses objectifs pédagogiques, en particulier dans des cours de langue où les expressions faciales jouent un rôle important dans l'apprentissage.

Au-delà des accommodements raisonnables, l'école peut aussi procéder à des ajustements volontaires ponctuels (culturels ou religieux) lorsqu'un conflit de normes se déclenche entre les parents d'un élève et l'école, mais qu'aucun droit fondamental n'est en jeu. Une telle situation pourrait survenir par exemple dans l'éventualité où un parent d'origine japonaise demanderait à un enseignant de français d'ajouter un roman japonais à la liste des livres de littérature étrangère entre lesquels les élèves doivent choisir leur programme de lecture. Dans une telle éventualité toutefois, les règles de sécurité et les droits d'autrui ne doivent évidemment pas être remis en cause. La « laïcité à la québécoise » constitue donc un cadre d'aménagement de la diversité et non un instrument d'effacement de la religion de l'espace public, qui s'applique à toutes les institutions sociales, y compris à l'école, et s'arrime logiquement à un projet d'éducation citoyenne fondé sur l'ouverture au pluralisme.

\section{Le rôle de l'école dans la formation des futurs citoyens}

Pour refléter adéquatement les orientations politiques et pédagogiques en matière de laïcité et d'ouverture à la diversité, l'école doit évidemment incorporer dans son curriculum formel (programmes formels, contenus enseignés), réel (interactions en classe) et caché (organisation de la vie scolaire), des dispositions cohérentes.

Même si certains regrettent le temps ou l'école véhiculait une « culture publique commune » définie dans les termes du groupe dominant, un certain consensus se forme depuis une quinzaine d'années autour de la nécessité de promouvoir un « vivre-ensemble » fondé sur l'égalité de tous les élèves. En effet, plusieurs critiques (Kymlicka, 1995 ; Taylor, 1992 ; Milot, 2002 : 155-161) ont dénoncé l'imposition d'un « arbitraire culturel », qui sous couvert de neutralité, véhiculait les valeurs et les normes de la culture majoritaire au détriment de celles des groupes minoritaires, ne serait-ce que quand les écoles du Québec étaient définies sur une base confessionnelle (jusqu'en 2000) (Groupe de travail sur la place de la religion à l'école, $1999: 174-178)^{6}$. Le vivre-ensemble s'est donc graduellement imposé comme l'emblème de la formation civique démocratique au Québec. Ce terme est d'ailleurs devenu une véritable marque déposée du ministère de l'Éducation, du

5 Notons toutefois que la jeune femme a déposé une plainte auprès de la Commission des Droits de la Personne et des Droits de la Jeunesse (CDPDJ) pour contester cette expulsion du centre de francisation.

6 Pour un aperçu plus détaillé du processus de déconfessionnalisation de l'école québécoise, consulter le texte de MILOT Micheline et TREMBLAY Stéphanie (2009) La religion dans le système scolaire public au Québec : un changement pour l'égalité et la diversité, Horizons-Projet de Recherche sur les politiques, 10 (2), pp. 34-39. 
Loisir et du Sport, qui en fait mention dans la plupart de ses énoncés politiques portant sur l'éducation. Or, ce vivre-ensemble sous-entend la capacité minimale des élèves à pouvoir s'ouvrir avec respect à des convictions morales différentes des leurs et à d'autres visions du monde. À cet égard, Milot propose, parmi plusieurs « vertus » civiques à développer en contexte scolaire, la « tolérance épistémique », une aptitude à « apprécier qu'une personne qui en arrive à des conclusions différentes de celles d'une autre ne peut, de ce fait, être taxée d'irrationalité. Dans la mesure où elles sont le fruit d'une réflexion rationnelle, elles sont dignes de respect » (cité dans le Groupe de travail sur la place de la religion à l'école, 1999 : 90).

On pourrait craindre qu'une telle forme de tolérance ne comprenne pas de limite et qu'elle ouvre la voie à un relativisme absolu. Mais une nuance s'impose. Dans une perspective relativiste, il serait en effet vain de chercher à délibérer et éventuellement à parvenir à des compromis puisque toutes les valeurs ou opinions se vaudraient entre elles. Toutefois, dans la proposition de Milot, il semble plutôt qu'il soit question de parvenir à un respect mutuel et à une base cognitive commune nécessaire à un dialogue ultérieur. En parlant plutôt des " raisons normativement modérées » échangées entre les partenaires d'une discussion (par exemple dans une classe), Weinstock (2000 : 414) confirme d'ailleurs cette nuance en spécifiant qu'il s'agit d'arguments que « toute personne raisonnable devra apprécier ou reconnaître comme valeur », même si elle n'endosse pas nécessairement cette valeur. Le développement d'une telle forme de tolérance apparaît donc intimement lié à la promotion d'une laïcité de « reconnaissance » en milieu scolaire.

En lien étroit avec cette aptitude d'ouverture « épistémique » à la diversité des convictions, le développement de l'autonomie et du jugement critique apparaît comme l'un des fondements clés de l'éducation libérale. En effet, pour assurer le bon fonctionnement de la démocratie et la participation entière des citoyens à la vie politique, les élèves doivent être aptes à prendre un certain recul par rapport à leurs idées préconçues et à se forger un point de vue éclairé sur différents enjeux. En ce sens, l'aptitude à la « délibération » (Gutmann, 1999 ; Weinstock, 2000 ; Groupe de travail sur la place de la religion à l'école, 1999 : 88-89), c'est-à-dire à l'échange régulé de points de vue argumentés plutôt que de simples opinions, doit se retrouver en contexte scolaire ${ }^{7}$. Nous verrons plus loin que cet objectif scolaire pose des défis de taille lorsqu'il entre en contradiction avec certaines convictions religieuses.

\section{La cohérence axiologique entre l'école et la famille}

Par ailleurs, si elle ne constitue pas le strict prolongement de la famille dans la perspective libérale, l'école ne doit pas non plus chercher à arracher l'enfant à ses racines familiales. Bien que la " socialisation primaire " des enfants en milieu familial s'harmonise assez souvent avec la " socialisation secondaire » transmise à l'école, il arrive parfois que ce lien n'aille pas de soi (Berger et Luckmann, 2003 ; Mead, 1963). C'est le cas entre autres avec les familles immigrées de fraîche date ou celles qui appartiennent à des groupes religieux marginaux (sectes, groupes fondamentalistes, etc.), dont la vision du

7 Cette compétence au dialogue démocratique est notamment développée dans le cadre du nouveau cours d'ECR dans les écoles du Québec. 
monde s'écarte de celle véhiculée à l'école. Dans de telles situations, l'élève peut alors se retrouver confronté à un choc des valeurs et ressentir une certaine confusion par rapport aux normes concurrentes mises de l'avant par sa famille et l'école. Une telle situation pourrait par exemple résulter d'une tension entre les valeurs féministes et individualistes du milieu scolaire et celles plus traditionnelles de la famille, ou encore entre la liberté accordée aux élèves à l'école et les règles strictes de la famille, en particulier à l'égard des relations amoureuses à l'adolescence.

Mais, selon plusieurs analystes, même si une attention constante est portée par les enseignants au respect des traditions de l'enfant, ce dernier tend graduellement à effectuer un tri entre les valeurs de l'école et celles de sa famille en s'élaborant une identité hybride entre les deux (Mc Andrew, 2008 : 8). Le rôle de l'école se limite donc, dans cette perspective, à veiller à ce que ce processus de négociation des valeurs ne bouleverse pas trop rapidement les repères de l'enfant en exerçant une « violence symbolique » à l'égard de l'univers normatif de sa famille. Pourtant, d'autres auteurs, surtout d'affiliation républicaine (Kintzler, 1998 ; Pena-Ruiz, 2001), sont d'avis que l'école doit préparer les élèves à acquérir des compétences civiques en induisant une certaine distance critique à l'égard de leur environnement familial.

Il reste qu'en psychologie, un consensus assez important met l'accent sur « l'histoire familiale d'une personne (notamment sa capacité à développer et à maintenir des liens d'attachement significatifs à ses parents) et sa capacité ultérieure de développer des sentiments d'empathie et d'altérité plus larges » (Mc Andrew, 2008 : 8). De surcroît, on peut logiquement s'attendre à ce qu'un enfant « reconnu » dans son environnement scolaire - au sens de Taylor (1992) - soit plus motivé et réussisse mieux à l'école.

L'ensemble de ces repères formels invite clairement l'école à prendre en compte la diversité religieuse et culturelle à l'école, mais jusqu'où et comment ? Il est difficile de fournir une réponse concrète et figée dans le temps à cette question puisque les balises évoluent sans cesse au gré de nouveaux compromis sociaux, mais nous tenterons d'esquisser, au fil de différents exemples empiriques et théoriques, la ligne directrice du rôle que devrait jouer la scolarisation dans le maintien ou la transformation des frontières ethniques au sein d'une telle approche.

\section{LES FONCTIONS DE LA SCOLARISATION ET LA CONSTRUC- TION DES FRONTIÈRES ETHNIQUES}

\section{La fonction d'instruction}

En ce qui a trait à la mission de transmission des savoirs, le premier enjeu consiste à déterminer jusqu'à quel point on peut transformer les savoirs scolaires pour y éliminer les biais ethnocentriques et y incorporer les perspectives de cultures minoritaires sans tomber dans l'écueil du relativisme absolu. Cette question se pose en particulier dans les écoles privées religieuses où les croyances religieuses deviennent le centre de gravité de l'enseignement ou chez des parents orthodoxes, dont les convictions religieuses 
peuvent entrer en tension avec le développement chez leurs enfants de la rationalité et du jugement critique. Mais, cet enjeu concerne aussi le cas de certaines minorités nationales, tels les Autochtones du Canada, qui revendiquent un contrôle de leurs propres institutions scolaires, y compris des contenus d'enseignement.

Comment départager un rapport au savoir différent, mais légitime en contexte pluraliste, d'un risque d'endoctrinement ? Et jusqu'où doit-on laisser les communautés trier les savoirs qu'elles transmettent aux futures générations afin de maintenir leurs identités distinctes?

Derrière la question de l'endoctrinement se posent d'abord les rapports entre religion et science, entre croyance et savoir. Dans les démocraties modernes, héritières des Lumières, on a longtemps supposé que la religion constituait un pendant de la méconnaissance et que plus la science permettrait de percer à jour les rouages de la connaissance, plus la religion s'en trouverait disqualifiée. Pourtant, force est de constater que la religion n'a pas disparu, mais qu'elle s'est transformée (Hervieu-Léger, 1999 : 13-27). Les croyances relèvent dorénavant plus de l'individu que des institutions religieuses et une liberté de choix a remplacé progressivement le conformisme aux traditions (Hervieu-Léger, 1999 : 53-60). Cela dit, l'appréhension de la « réalité objective » passe essentiellement par le prisme de la rationalité scientifique, qui détient, dans les démocraties modernes, «le monopole du savoir légitime », pour paraphraser Weber. Dans ce paradigme, seuls les faits matériels et visibles dans l'espace et dans le temps relèvent du savoir légitime : «Pour lui les tables et les pommes existent, mais pas le père Noël et les licornes qui n'existent pas, qui sont des fictions » (Grondin, 2009 : 9). Dans ce contexte, la religion qui se fonde sur de l'invisible, qui n'est pas falsifiable et se dérobe à la preuve empirique, n'est associée qu'à un pari ou une hypothèse et n'est pas reconnue comme une forme de savoir valide (Hervieu-Léger, 1999 : 30-31).

Appliquée au contexte scolaire, cette logique n'admet aucun autre rapport au savoir que celui qui relève de la connaissance scientifique, falsifiable et donc susceptible d'évoluer dans le temps au fil des nouvelles découvertes. Toutefois, certains parents récusent ce "monopole » de la science à l'école et considèrent qu'elle relève d'une tentative d'endoctrinement à un humanisme séculier, une idéologie aussi dogmatique que n'importe quelle conception religieuse radicale (Zine, 2008 : 11). Les tenants de ce point de vue, qui plaident aussi, à l'instar des communautariens, pour une autorité prépondérante des parents sur l'éducation scolaire, font donc valoir la relativité du savoir scientifique par rapport aux croyances religieuses. Une telle reconnaissance des limites de la science a certes été reconnue par d'éminents philosophes des sciences, comme Kuhn, qui affirmait qu'au fondement de tout paradigme scientifique, on trouvait un amas hétéroclite de croyances non démontrables (Kuhn, 1972).

Mais, une telle critique de la conception scientifique fondant la légitimité des savoirs scolaires semble négliger un aspect fondamental : le type de rationalité différent qui sous-tend la science et la religion. La socialisation scolaire orientée vers certaines croyances religieuses tend en effet à s'aligner sur une "rationalité en valeur » au sens de Weber, selon laquelle les convictions deviennent la finalité absolue de toute action sociale. Malgré l'impact important des sentiments (irrationnels) dans ce type de conduite, 
Weber considère qu'elle relève de l'action rationnelle en ce qu'elle essaie de se donner une cohérence interne se traduisant par des actes conformes à la conviction (Freund, 1983 : 93). L'individu guidé par la rationalité en valeur se voit donc entièrement mu par la motivation de correspondre à un idéal défini par une ou plusieurs valeurs ultimes. Mais ce faisant, il risque de mal évaluer les conséquences réelles de son comportement et de glisser dans l'irrationalité et alors, explique Derocher, en parlant des dérives sectaires, « La rationalité risque de perdre de la force et l'irrationalité, de prendre le dessus » (2008: 46). À la différence de la religion, la science repose sur une " rationalité en finalité » qui juge de l'action selon l'adéquation entre les résultats poursuivis et les ressources mobilisées pour l'obtenir. Elle prend donc en considération les conséquences prévisibles de l'activité et repose sur une méthode (scientifique) qui lui permet de suivre un protocole défini et d'exercer un certain contrôle sur les variables qu'elle souhaite examiner.

En contexte scolaire, deux éléments fondamentaux permettent donc de distinguer la science et la religion et de légitimer la suprématie de la première approche au détriment de l'autre. D'abord, même si l'on admet que la science s'appuie sur un certain nombre de postulats indémontrables, elle s'autocritique continuellement et évolue au fil du temps, ce qui n'est pas forcément le cas des croyances religieuses (surtout conservatrices ou fondamentalistes), qui peuvent être relativement figées dans le temps et même viser un retour en arrière (Hervieu-Léger, 1999 : 29-32 ; Roy, 2002). En outre, puisque la conception religieuse s'appuie sur une rationalité en valeur, parfois située à la frange de l'irrationalité, une scolarisation fondée sur une telle vision du monde risquerait de former des « croyants marginaux » mésadaptés aux exigences de la société moderne et démocratique dans laquelle ils se trouvent (McClosky et Schaar, $1965: 22$ ).

En effet, une conception élastique des savoirs scolaires, que ce soit par un mélange des genres scientifique et religieux ou par une surreprésentation des cultures et des langues minoritaires (hétérocentrisme égalitaire), exposerait nécessairement l'école à un risque de relativisation de la connaissance légitime et pourrait provoquer un état d'anomie. Les élèves qui n'auraient pas été socialisés par le biais de l'école aux compétences et aux savoirs nécessaires à leur intégration dans une démocratie moderne (règles, normes, rôles sociaux, vertus civiques, etc.) pourraient facilement se percevoir comme des étrangers à cette société et tomber entre les mailles du filet social : "L'individu anomique n'a plus le sens de la cohésion sociale, il vit dans l'instant, sans attache avec les autres et a perdu l'unité dynamique de sa personnalité » (Rivest, 1994 : 11). Cet état reflète « une désintégration du sens de l'attachement à la société et d'un sentiment de solitude intense » (Rivest, 1994 : 14). C'est entre autres pour éviter ce risque et favoriser le développement d'une « compétence sociale » commune, nécessaire au maintien de la démocratie, que l'État a le devoir, selon plusieurs auteurs, de fixer un certain nombre de matières scolaires (scientifiques, techniques et culturelles) obligatoires pour tous les élèves (Bourgeault et al., 1995 : 94). De plus, la mise en contact des enfants avec d'autres points de vue et valeurs que celles émanant du milieu familial constitue l'une des conditions pour que l'État puisse garantir l'indépendance morale des individus dans l'approche libérale et leur offre la possibilité de réviser leurs convictions (Groupe de travail sur la place de la religion à l'école, $1999:$ 57-60 et 87-93). 
Le litige juridique déclenché récemment au Québec par deux parents, ayant demandé au tribunal le droit d'exempter leur enfant du cours d'éthique et culture religieuse $(\mathrm{ECR})^{8}$ au nom de leur liberté de conscience et de religion, exprime bien la tension entre deux conceptions normatives de la mission d'instruction de l'école. Or, dans cette cause traitée en juin 2009, le juge Dubois a clairement établi que le cours ECR ne brimait pas la liberté de conscience et de religion dans la mesure où « l'on [y] fait une présentation globale de diverses religions sans obliger les enfants à y adhérer $»$. Toutefois, un autre jugement, déposé en juin 2010 (mais déjà porté en appel par le gouvernement), entre précisément en contradiction avec celui de l'année dernière. En effet, ce nouveau jugement a exempté le collège Loyola High School de l'obligation de dispenser le cours ECR à condition qu'il le remplace par un cours « équivalent », plus compatible avec ses valeurs catholiques. En plus d'invoquer à titre d'argument la suprématie de Dieu dans la Constitution canadienne, le juge Dugré écrit : « [L]'obligation imposée à Loyola d'enseigner la matière obligatoire ECR de façon laïque revêt un caractère totalitaire qui équivaut, essentiellement, à l'ordre donné à Galilée par l'Inquisition de renier la cosmologie de Copernic $»^{10}$. Poussant plus loin la logique de la « reconnaissance » démocratique, ce dernier jugement illustre que le débat sur la prise en compte de la diversité dans le curriculum et le rôle de l'école dans la formation civique des enfants est loin d'être clos, même dans la sphère juridique !

\section{La fonction de socialisation}

Si l'État doit baliser la transmission des savoirs à l'école de manière à équilibrer une certaine représentation du pluralisme avec le souci d'instruire à un savoir relativement objectif, on peut se demander si une telle reconnaissance de la diversité doit inclure le droit des minorités de gérer leurs propres écoles à même les fonds publics.

Selon Kymlicka (1995) et d'autres, dont Mc Andrew (2000), une ligne de démarcation doit être tracée entre les minorités nationales et les minorités issues de l'immigration quant à leur droit d'assurer la reproduction culturelle de leur communauté à travers l'école. Si les premières ont été soumises contre leur gré à une colonisation, à des pratiques esclavagistes et à des " génocides culturels » dans le passé, les autres sont arrivées sur une base volontaire et individuelle dans la société d'accueil (Mc Andrew, 2000 ; Gutmann, 1999). Selon ces auteurs, il serait donc légitime d'accorder plus de latitude aux minorités nationales (comme les Autochtones du Canada), dans l'orientation des contenus éducatifs de l'école (par exemple, l'enseignement des langues d'origine), notamment pour assurer une forme de réparation historique et une pérennité de leur patrimoine culturel. L'argument de la survie culturelle des minorités nationales, comme s'il s'agissait de « baleines » ${ }^{11}$ en voie d'extinction, nous semble toutefois une arme à deux tranchants, car s'il conforte

8 Un cours d'enseignement sur les religions (non confessionnel) entré en vigueur en septembre 2008, obligatoire dans toutes les écoles du Québec.

9 Suzanne Lavallée et Daniel Jutras c. Commission scolaire Des Chênes [2009] 405-17-000946-082 (C.S. Qué.) parag. 69.

10 Loyola High School et John Zucchi c. Michelle Courchesne en sa qualité de ministre de l'Éducation, du Loisir et du Sport [2010] 500-17-045278-085 (C.S. Qué.) parag. 331.

11 Pour paraphraser l'expression provocatrice de Habermas à l'endroit des Québécois : « Les Québécois ne sont pas des baleines » (Renaut et Touraine, 2005 : 111). 
l'idée légitime de leur reconnaître des droits culturels, il risque potentiellement de les enfermer dans une image figée et folklorisée de leur culture, qui ne coïncide peut-être pas avec ses transformations réelles.

On peut aussi se questionner sur la pertinence d'une ligne trop rigide entre les minorités nationales et les immigrants quant à la prise en compte des identités groupales à l'école. Comment classer en effet les nations «sans État », comme la communauté juive, qui possède aussi un patrimoine culturel fragile et qui constitue une communauté de longue date dans plusieurs pays, dont au Canada, sans pour autant faire l'objet d'une minorité nationale ? Il nous semble qu'un tel modèle de " citoyenneté multiculturelle ", quoique fort utile, doit laisser place à une certaine souplesse pour s'adapter à des cas de figure différents. Dans une telle zone grise, à mi-chemin entre le cas des minorités nationales et celui des immigrants récents, dont les sociétés d'accueil souhaitent éviter la ghettoïsation, s'est entre autres posé celui des Amish aux États-Unis. Dans ce cas, le tribunal a considéré légitime la demande des Amish de gérer l'offre scolaire destinée aux enfants de sa communauté, dans la mesure où cette dernière n'était pas « opposée aux valeurs américaines » (cité dans Mc Andrew, 2000 : 30). L'État a toutefois préservé le droit des enfants de quitter librement la communauté en imposant un cours d'éducation civique, une fois par semaine, dispensé par un employé de l'extérieur de la communauté. Ce garde-fou établi par l'État constitue donc, même dans un contexte aussi libéral qu'aux États-Unis, une mesure minimale pour assurer l'indépendance morale des individus (des enfants) et leur octroyer des droits propres, distincts de ceux de leurs parents.

Étant donné l'ampleur du mandat de socialisation de l'école, il va de soi que l'un des lieux névralgiques de la prise en compte du pluralisme et des cultures minoritaires réside dans le cas des écoles ethnoreligieuses (financées par l'État). De telles institutions scolaires, rattachées structurellement ou symboliquement à une culture ou à une religion particulière, présentent en effet pour les uns des voies d'articulation souhaitables entre les exigences de la citoyenneté et le maintien des identités communautaires et pour les autres, des lieux de ghettoïsation réfractaires aux valeurs libérales. La principale critique qui surgit à l'égard de ces écoles concerne les faiblesses du curriculum réel (interactions entre les élèves), dans la mesure où l'environnement scolaire de ces institutions forme généralement une " enclave " homogène plus ou moins encapsulée autour d'une culture ou d'une religion particulière. La plupart des élèves et des enseignants appartiennent en effet à la communauté chapeautée par l'école et selon plusieurs, cette concentration ethnique ou religieuse peut provoquer un repli politique, social et culturel de la communauté sur elle-même et un déficit dans la formation civique des élèves.

À cet égard, plusieurs auteurs, dont Gutmann $(1995 ; 1999)$ et Milot (2005), ont cerné les conditions nécessaires à l'établissement d'une " éducation démocratique » en contexte scolaire. Selon la première, la préparation des jeunes à la « reproduction consciente de leur société » (Gutmann, 1999 : 42) (participation aux débats publics, vote aux élections, lecture critique des enjeux de société, etc.) se trouve intrinsèquement liée à la « délibération démocratique » et à l'apprentissage de l'ouverture aux différences. Or, un tel laboratoire éducatif ne peut se développer que dans un environnement pluraliste, à l'image de la société, et favorable à une libre remise en question des idées reçues. C'est pourquoi plusieurs détracteurs des écoles séparées craignent que ces institutions provo- 
quent une « balkanisation » du système d'éducation et nuisent à l'intégration sociale en contexte pluraliste (Gutmann, 1995). Ils estiment en effet qu'une exposition précoce des élèves à une diversité de points de vue et d'options morales constitue une condition indispensable à l'apprentissage de la tolérance et du civisme (Macedo, 1995). Gutmann ajoute que les écoles ethniques ou religieuses, telles que les écoles afrocentristes, prôneraient une perspective " séparatiste » générant un sentiment de méfiance à l'égard de l'altérité en enseignant aux élèves « le sens de la supériorité raciale» (1995: 557-558).

En revanche, selon ses partisans (Thiessen, 2001 ; Zine, 2008), l'intériorisation des compétences civiques ne dépend pas tant de la fréquentation d'institutions scolaires communes, qui se révèlent souvent culturellement homogènes (par exemple en banlieue des grandes villes), que des valeurs éducatives et des approches pédagogiques mises en place. Selon Thiessen, un environnement scolaire plus homogène sur le plan ethnique ou religieux favoriserait même un apprentissage plus efficace des vertus civiques libérales (comme l'aptitude à la délibération et à l'ouverture à l'altérité) qu'un milieu pluraliste, puisque ce cadre plus restreint épouserait davantage la «culture primaire » des enfants (2001 : 29-43). Une telle hypothèse semble confortée par quelques études empiriques, dont celle de Sweet (1997), dans laquelle une mère interrogée décrit de manière exemplaire le paradoxe de l'ouverture à l'autre dans le contexte d'une école juive de Toronto où elle a envoyé sa fille : «And here I am, educating my child separatly. But there's a paradox [...] The more I teach her who she is, then the better she can live with others » (1997: 112). Ainsi, la socialisation scolaire «marginale », articulée autour d'une tradition religieuse ou culturelle particulière, réduirait ainsi, selon ses promoteurs, le hiatus possible entre les valeurs familiales et celles de l'école publique de manière à permettre aux enfants de développer une identité sociale plus forte et plus positive (Thiessen, 2001 ; Zine, 2008). Zine reconnaît cependant que la discipline stricte, les rapports hiérarchiques et les méthodes pédagogiques centrées sur des structures de savoirs traditionnels et encyclopédiques laissent souvent peu de place aux interactions entre les élèves et au dialogue dans plusieurs écoles musulmanes torontoises (2008: 316).

Mais qu'en est-il de l'intégration sociale effective des élèves fréquentant de telles écoles ? Peu d'études ont abordé de front cette question, mais un chercheur québécois (Sercia, 2009) a récemment observé que les élèves fréquentant des écoles ethnoreligieuses (arméniennes, juives et musulmanes) à Montréal développaient généralement un sentiment d'appartenance élevé à la fois vis-à-vis leur groupe d'origine et la société d'accueil. Cette même étude a toutefois permis d'établir un lien entre la concentration ethnique caractérisant les écoles ethnoreligieuses et le degré plus faible d'ouverture des jeunes au pluralisme culturel et aux autres groupes ethniques. Un constat similaire se dégageait d'ailleurs d'une étude menée en 1999 (Mc Andrew et al.) qui avait démontré que la densité ethnique (élèves issus de l'immigration) au sein des écoles montréalaises affectait positivement l'ouverture à la diversité culturelle.

\section{La fonction de qualification}

Si la prise en compte de la diversité culturelle pose des questions épineuses au regard des mandats d'instruction et de socialisation de l'école, elle interpelle aussi tout particulièrement sa mission de qualification, même si a priori le lien entre ethnicité et 
cheminement scolaire peut paraître plus nébuleux. Certes, la fonction de qualification (et de sélection) de l'école dans les démocraties libérales se fonde sur le principe d'égalité des chances, sa mise en œuvre sur le terrain ne va pas toujours de soi. En effet, le débat ne se focalise plus autant sur l'accès à la scolarisation obligatoire et les cheminements différentiels, qui s'actualisent surtout à travers les choix de carrière aux niveaux collégial et universitaire, que dans la « discrimination éducative » entre les différents groupes (Mc Andrew, 2000 : 18). Plusieurs études révèlent en effet l'existence de différences marquées dans les résultats et les parcours scolaires de groupes majoritaires et minoritaires et il semblerait qu'un "résiduel ethnique » rende compte dans une certaine mesure de ces écarts. Mais contrairement à ce que prétend souvent le sens commun, cet effet ne jouerait pas systématiquement en faveur des majorités. C'est notamment le cas de plusieurs élèves d'origine asiatique (entre autres les locuteurs du chinois) qui affichent une surperformance scolaire par rapport aux élèves du groupe majoritaire dans le contexte québécois (Girouard, 2010).

Ici encore, un certain clivage se dessine entre les profils de réussite scolaire des minorités nationales et des immigrants plus récents. En contexte migratoire, les nouveaux arrivants tendent en effet à s'intégrer à la société sur une base individuelle et à développer des stratégies de mobilité sociale en conséquence, qui passent notamment par l'école. Plusieurs auteurs, dont Valet et Caille (2000), ont étudié les trajectoires scolaires d'enfants d'immigrants pour constater que, dans plusieurs cas, les fortes aspirations éducatives des parents les poussent à mobiliser plus de ressources vers le projet scolaire de leurs enfants que chez les parents du groupe majoritaire. Ceux-ci voient en effet l'école comme le tremplin par excellence vers une mobilité sociale ascendante, et c'est pourquoi ils sont prêts à réaliser d'importants sacrifices pour supporter la réussite de leurs enfants. Ces stratégies de résilience familiales n'empêchent cependant pas qu'une forme plus voilée de discrimination imbibe parfois le curriculum caché ou implicite de l'école, pouvant par le fait même nuire à la « réussite éducative » de certains groupes, entre autres ceux dont les identités religieuses s'expriment par des signes vestimentaires ostensibles. La question de la religion frappe en effet l'imaginaire collectif dans de nombreuses sociétés sécularisées d'Amérique du Nord et d'Europe, où le religieux « fort » est encore implicitement associé à l'obscurantisme, mais où en même temps une religion patrimoniale demeure toujours la matrice symbolique de la culture dominante.

Cependant, en ce qui concerne le cas des minorités nationales (involontaires), la problématique de la sélection scolaire prend une tournure plus dramatique. En effet, qu'il s'agisse d'Autochtones ayant été soumis à la colonisation ou de groupes issus de l'esclavage, le passé trouble et la mémoire collective d'opprimé de ces groupes continuent souvent de saborder leur rapport à l'École. Un peu partout dans le monde, les minorités nationales accusent d'ailleurs souvent un net retard scolaire, en comparaison aux autres groupes, auquel s'ajoutent d'autres problèmes d'intégration sociale (alcool, violence conjugale, décrochage, suicide, etc.) plus ou moins liés à la déstructuration historique de leurs communautés. Selon Ogbu (1982), cet écart culturel entre les minorités nationales et l'institution scolaire relèverait d'une " discontinuité culturelle secondaire », provoquée par une mise en contact des groupes majoritaires et minoritaires. Thésée et Carr expliquent : " Cette forme de discontinuité culturelle se cristallise dans le système scolaire, sorte de microcosme de la société où se déploie la culture dominante, et l'éducation étant 
le véhicule privilégié de changement social, ce qui s'y passe est relayé à travers toute la communauté ») (2007: 156).

Or, cette forme de dissonance entre certains groupes dominés et l'institution scolaire rejaillit sur tous les lieux d'intégration sociale et peut s'exprimer entre autres par des barrières à l'emploi, du profilage racial, des attitudes racistes dans les médias et l'opinion publique. Ces mécanismes explicites ou subtils de racisation s'appuient pourtant généralement sur des idéologies légitimatrices qui associent les obstacles vécus par ces communautés à des conséquences de leurs traits de personnalité ou de leurs capacités individuelles. Or, cette discrimination éducative bien réelle peut exacerber chez les minorités nationales une attitude pessimiste à l'égard de l'école, qui se transforme alors à leurs yeux en " corps étranger ", mais qui représente en même temps le principal véhicule d'émancipation et de mobilité sociale (Thésée et Carr, 2007). À cet égard, l'épisode de l'assimilation forcée de nombreux Autochtones canadiens dans des pensionnats indiens au cours des années 1950-1960 a beaucoup nui en envenimant à long terme les rapports des Autochtones à l'école. La discrimination en éducation dirigée vers les minorités nationales peut en outre entraîner des stratégies identitaires collectives orientées vers la survivance, la résistance et l'opposition au reste de la société, selon Thésée et Carr (2007 : 159).

La théorie de la résistance a d'abord été élaborée dans l'univers économique par Willis pour traiter des modalités d'opposition de la classe ouvrière à l'École. Selon ce sociologue, la " "culture anti-école" fournit de puissants critères implicites et des processus contraignants d'expérience qui amènent les jeunes de la classe ouvrière à faire le choix “volontaire" d'entrer à l'usine et ainsi à aider à la reproduction [...] de la structure de classe de l'emploi » (Willis, 1978 : 51). Autrement dit, les jeunes issus de la classe ouvrière procèdent en quelque sorte à une « inversion du stigmate » (Goffman, 1975) qui leur est accolé (appartenir à la classe ouvrière) de manière à développer une signification positive à la reproduction de leurs conditions de vie. Or, un processus similaire touche plusieurs minorités nationales, tels les jeunes Noirs de Montréal (notamment la communauté haïtienne) et de Toronto, qui tendent à résister et à s'opposer à la société en général dont ils se sentent exclus et marginalisés en développant un rapport négatif à l'institution scolaire. Thésée et Carr précisent qu' « ils [jeunes Noirs] considèrent l'expérience scolaire en soi comme un insidieux processus d'acculturation, c'est-à-dire, un processus à l'issue duquel ils penseront, se comporteront et seront comme les Blancs » (2007 : 161). C'est entre autres la construction de cette nouvelle frontière symbolique entre certains jeunes Noirs et le reste de la société qui éclaire le développement de l' " Ebonics ", un langage vernaculaire mis au point par certains Afro-américains afin de se parler sans être compris des « Blancs ». Il en va de même pour les « Beurs », descendants d'émigrés d'Afrique du Nord et nés en France, qui communiquent en « verlan » (l'envers des mots) entre eux pour « résister » à la société française et consolider leur identité collective.

Parfois, l'émergence d'identités de résistance ou d'opposition peut conduire certains groupes à envisager une stratégie collective de « séparation » à l'égard du reste de la société, pour reprendre les catégories d'acculturation de Berry (2005), une stratégie qui dans la sphère éducative peut se traduire par le choix d'une école privée, ethnoreligieuse. C'est notamment le cas des écoles afrocentristes, dont la popularité grandissante a même donné lieu à l'ouverture d'une première institution de ce genre à Toronto, en 2009. Visant 
à diminuer le taux de décrochage scolaire chez les jeunes Noirs, la direction de cette nouvelle école affirme respecter le programme scolaire du ministère de l'Éducation de l'Ontario, mais utilise des ressources culturelles (centrées sur la culture noire) différentes pour poursuivre les mêmes objectifs académiques. Si certains voient d'un mauvais œil l'émergence de ce type d'écoles, dont ils redoutent le potentiel de fragmentation sociale (Gutmann, 1995), la réalité nous montre qu'il peut s'agir d'une avenue intéressante pour juguler les échecs scolaires importants de la communauté et promouvoir une meilleure égalité devant l'éducation.

\section{CONCLUSION}

Les demandes de prise en compte de la diversité religieuse et culturelle dans l'espace scolaire, comme nous croyons l'avoir illustré, interpellent l'État à divers niveaux et mettent en tension plusieurs valeurs, dont le pluralisme, l'égalité, les droits collectifs et le bien commun. Or, étant donné l'ampleur de ses mandats d'instruction, de socialisation et de qualification des futurs citoyens et parce qu'elle se situe à la charnière de l'espace public et de l'espace privé, l'école ne peut « gérer la diversité » à la manière de n'importe quelle autre institution. À la jonction du mandat d'instruction et de socialisation de l'école, la socialisation scolaire « marginale », dont les écoles ethnoreligieuses constituent un cas de figure exemplaire, a notamment surgi comme un enjeu de taille s'imposant aux démocraties libérales. En ce sens, il conviendrait d'approfondir les études empiriques portant sur le curriculum formel et réel de ce type d'institutions scolaires. Ce registre de la recherche affleure dans les écoles musulmanes, mais peu dans les autres, comme les écoles juives, pourtant plus nombreuses en Europe et en Amérique du Nord et implantées depuis plus longtemps. D'ailleurs, une comparaison entre les écoles juives et musulmanes au regard de leurs éthos spécifiques et de leur relation à la citoyenneté se révélerait tout à fait pertinente.

Finalement, même s'il est difficile de démêler l'écheveau des divers facteurs entrant en jeu dans la genèse des inégalités scolaires et de la fonction de sélection de l'École, plusieurs auteurs ont montré l'effet significatif de la variable ethnique qui jouerait à des niveaux différents selon la nature des groupes. La plupart des études révèlent en effet que si les immigrants réussissent souvent à développer des stratégies de résilience scolaire efficaces (Valet et Caille, 2000), les minorités nationales ne parviennent pas aussi facilement à franchir les barrières systémiques qui se dressent sur leur parcours académique. C'est pourquoi plusieurs auteurs se montrent plus ouverts à une intervention spécifique de l'État dans le cas de ces communautés. Mais, il reste qu'à notre sens, il importe de ne pas verrouiller une fois pour toutes l'offre de programmes compensatoires afin de tenir compte des besoins spécifiques des différents groupes d'élèves (minoritaires ou non), en particulier ceux plus ciblés par le racisme.

S'il n'y a pas de réponse définitive à la question du rôle de la scolarisation dans le maintien ou la transformation des frontières ethniques, l'analyse des trois grandes fonctions de l'École en contexte démocratique en général et libéral en particulier nous a toutefois mis sur la piste de repères essentiels. Dans la perspective de l'indépendance morale des individus, pierre angulaire du projet libéral, un certain équilibre s'impose d'abord entre 
les droits des parents, de l'État et des enfants, et le respect des convictions doit, dans la mesure du possible, épouser la volonté de l'enfant. Afin de respecter le fonctionnement du régime démocratique et les droits fondamentaux de la personne, une attention particulière doit en outre être accordée au développement des « vertus » civiques à l'école. Or, ces dispositions de caractère impliquent non seulement que l'élève soit plongé dans un environnement non sécurisé par rapport à l'univers familial, mais qu'il possède la liberté de réviser ses propres convictions s'il le souhaite.

\section{Références bibliographiques}

BARTH Fredrik (1969) Ethnic Groups and Boundaries, Boston, Little, Brown and Co, 153 p.

BERGER Peter et LUCKMANN Thomas (2003) La construction sociale de la réalité (traduit de l'américain par P. Taminiaux), Paris, Armand Colin (1 ère éd. 1966), 357 p.

BERRY John (2005) Acculturation: Living successfully in two cultures, International Journal of Intercultural Relations, 29, pp. 697-712.

BOSSET Pierre (2007) Les fondements juridiques et l'évolution de l'obligation d'accommodement raisonnable, in Myriam Jézéquiel Éd., Les accommodements raisonnables : quoi, comment, jusqu'où? Des outils pour tous, Cowansville, Éditions Yvon Blais, pp. 3-28.

BOURGEAULT Guy, GAGNON France, McANDREW Marie et PAGÉ Michel (1995) L'espace de la diversité culturelle et religieuse à l'école dans une démocratie de tradition libérale, Revue Européenne des Migrations Internationales, 11 (3), pp. 79-103.

COMMISSION DE CONSULTATION SUR LES PRATIQUES D'ACCOMMODEMENT RELIÉES AUX DIFFÉRENCES CULTURELLES (2007) Fonder l'avenir. Le temps de la conciliation, Rapport rédigé par Gérard Bouchard et Charles Taylor, Québec, Gouvernement du Québec, 310 p.

COMMISSION DES DROITS DE LA PERSONNE ET DES DROITS DE LA JEUNESSE (CDPDJ) (1995) Le Québec face au pluralisme religieux : un défi d'éthique sociale, Montréal, Gouvernement du Québec, $30 \mathrm{p}$.

DEI George (1994) Reflections of an anti-racist pedagogue, in Lorna Erwin et David MacLennan Eds., Sociology of Education in Canada: Critical Perspectives on Theory, Research, and Practice, Toronto, Copp Clark Longman Ltd, pp. 290-310.

DEROCHER Lorraine (2008) Vivre son enfance au sein d'une secte religieuse, Montréal, Les Presses de l'Université du Québec, 183 p.

FREUND Julien (1983) Sociologie de Max Weber, Paris, Presses Universitaires de France, 257 p.

GIROUARD Catherine (2010) Les immigrants québécois et les études, un exemple de résilience (entrevue avec Marie Mc Andrew), Journal Métro, 20 janvier 2010, [en ligne] consulté le 27 septembre 2011. URL : http://www.journalmetro.com/carrieres/article/427387--les-immigrantsquebecois-et-les-etudes-un-exemple-de-resilience

GLAZER Nathan and MOYNIHAN Daniel Patrick (1976) Ethnicity: Theory and experience, Cambridge, Harvard University Press, 531 p.

GOFFMAN Erving (1975) Stigmate. Les usages sociaux des handicaps, Paris, Les éditions de Minuit, $175 \mathrm{p}$.

GRONDIN Jean (2009) La philosophie de la religion, Paris, Presses Universitaires de France, 127 p.

GROUPE DE TRAVAIL SUR LA RELIGION À L'ÉCOLE (1999) Laïcité et religions. Perspective nouvelle pour l'école québécoise, Québec, gouvernement du Québec, 282 p.

GUTMANN Amy (1999) Democratic education, New Jersey, Princeton University press $\left(1^{\text {st }}\right.$ ed. 1987), $348 \mathrm{p}$. 
GUTMANN Amy (1995) Civil Education and Social Diversity, Ethics, 105, pp. 557-579.

HERVIEU-LÉGER Danièle (1999) Le pèlerin et le converti. La religion en mouvement, Paris, Flammarion, $289 \mathrm{p}$.

JUTEAU Danielle (1999) L'ethnicité et ses frontières, Montréal, Les Presses de l'Université de Montréal, $226 \mathrm{p}$.

KINTZLER Catherine (1998) Tolérance et laïcité, Paris, Saint-Sébastien-sur-Loire Pleins feux, $81 \mathrm{p}$.

KUHN Thomas (1972) La structure des révolutions scientifiques, Paris, Flammarion, 284 p.

KYMLICKA Will (1995) La citoyenneté multiculturelle, Montréal, Boréal, 358 p.

LEROUX Georges (2010) L'État doit viser à déstabiliser les systèmes absolutistes de croyances, Pour une école libre, [en ligne] consulté le 26 août 2010. URL : http://www.republiquedebananes.com/2010/05/18/george-leroux\%C2\%A0-l'etat-doit-viser-a-destabiliser-les-systemesabsolutistes-de-croyance/

MACEDO Stephen (1995) Liberal Civic Education vs Religious Fundamentalism. The Case of God vs John Rawls?, Ethics, 105 (3), pp. 468-496.

MC ANDREW Marie (2008) Module de formation à l'intention des gestionnaires. La prise en compte de la diversité culturelle et religieuse en milieu scolaire : de la théorie à la pratique, Québec, gouvernement du Québec, ministère de l'Éducation, du Loisir et du Sport, 143 p.

MC ANDREW Marie (2000) Relations ethniques, pluralisme et égalité : le rôle de l'école, Leçon inaugurale déposée au Département d'études en éducation et d'administration de l'éducation, Université de Montréal, $39 \mathrm{p}$.

MC ANDREW Marie, MILOT Micheline, IMBEAULT Jean-Sébastien et EID Paul (2009) L'accommodement raisonnable et la diversité religieuse à l'école publique. Normes et pratiques, Montréal, Fides, 295 p.

MC ANDREW Marie, PAGÉ Michel, JODOIN Mathieu et LEMIRE Francine (1999) Densité ethnique et intégration des élèves d'origine immigrante au Québec, Études ethniques au Canada, 31 (1), [en ligne] consulté le $1^{\text {er }}$ septembre 2011. URL : http://findarticles.com/p/articles/mi hb039/is_1_31/ai_n28756457/?tag=mantle_skin;content

MC CLOSKY ${ }^{-}$Herbert and SCHAAR John H. (1965) Psychological Dimensions of Anomy, American Sociological Review, 30 (1), pp. 14-40.

MEAD George Herbert (2006) L'esprit, le soi et la société (traduit par J. Cazeneuve, E. Kaelin et G. Thibault), Paris, Presses Universitaires de France (1 ère éd. 1963), 434 p.

MILOT Micheline (2008) La laïcité, Montréal, Novalis, 118 p.

MILOT Micheline (2005) Tolérance, réciprocité et civisme : les exigences des sociétés pluralistes, in Fernand Ouellet Éd., Quelle formation pour l'éducation à la religion, Québec, Les Presses de l’Université Laval, pp. 11-32.

MILOT Micheline (2002) Laïcité dans le Nouveau Monde. Le cas du Québec, Paris, Turnhout, Brepols Publishers, $177 \mathrm{p}$.

MILOT Micheline et ESTIVALÈZES Mireille (2008) La prise en compte de la diversité religieuse dans l'enseignement scolaire en France et au Québec, Éducation et Francophonie, 36 (1), pp. 86-102.

MINISTÈRE DE L'ÉDUCATION, DU LOISIR ET DU SPORT (2008) Éthique et culture religieuse (version révisée), Programme du premier cycle et du deuxième cycle du secondaire, Québec, gouvernement du Québec, $86 \mathrm{p}$.

MINISTĖRE DES COMMUNAUTÉS CULTURELLES ET DE L'IMMIGRATION (1990) Au Québec pour bâtir ensemble, Énoncé de politique en matière d'immigration et d'intégration, Montréal, Direction des communications, $112 \mathrm{p}$.

NOVAK Michael (1971) The Rise of Unmeltable Ethnics, London, Macmillan Pub Co, 376 p.

PENA-RUIZ Henri (2001) Dieu et Marianne : philosophie de la laïcité, Paris, Presses Universitaires de France, 360 p. 
POTVIN Maryse et CARR Paul (2008) La valeur ajoutée de l'éducation antiraciste : conceptualisation et mise en œuvre au Québec et en Ontario, Éducation et Francophonie, 36 (1), pp. 197-217. OGBU John (1982) Cultural discontinuities and schooling, Anthropology and Education Quarterly, 13 (4), pp. 290-307.

RIVEST Jean (1994) Anomie et délinquance chez un groupe d'adolescents anglais de la région métropolitaine de Montréal, Mémoire de maîtrise inédit, Université du Québec à Montréal, 81 p. ROY Olivier (2002) L'islam mondialisé, Paris, Édition du Seuil, 234 p.

SERCIA Pierre (2009) L'intégration sociale des élèves fréquentant les écoles ethnoreligieuses de l'île de Montréal, Thèse de doctorat inédite, Université de Montréal, 252 p.

STOLZENBERG Naomi (1993) He drew a circle that shut me out: assimilation, indoctrination, and the paradox of liberal education, Harvard Law Review, 106 (3), pp. 588-666.

SWEET Lois (1997) God in The Classroom: The Controversial Issue of Religion in Canada's Schools, Toronto, McClelland and Stewart, $288 \mathrm{p}$.

TABOELDA-LEONETTI Isabelle (1990) Stratégies identitaires et minorités. Le point de vue du sociologue, in Carmel Camilleri, Joseph Kastersztein, Edmond Marc Lipansky, Hanna Malewska-Peyre, Isabelle Taboada-Leonetti et Ana Vasquez Éds., Stratégies identitaires, Paris, Presses Universitaires de France, pp. 43-84.

TAYLOR Charles (1992) Multiculturalism and "The Politics of Recognition", Paris, Flammarion, $175 \mathrm{p}$.

THÉSÉE Gina et CARR Paul (2007) Les mesures d'équité et les discontinuités culturelles, in Claudie Solar et Fasal Kanouté Éds., Questions d'équité en éducation et formation, Montréal, Éditions Nouvelles, pp. 143-172.

THIESSEN Elmer J. (2001) In defence of religious schools and colleges, Montréal, McGill-Quenn's University Press, 368 p.

VALET Louis-André et CAILLE Jean-Paul (2000) La scolarité des enfants d'immigrés, in Agnès Van Zante Éd., L'école, état des savoirs, Paris, La découverte, pp. 293-300.

WEBER Max (1995) Les catégories de la sociologie, T. 1 Économie et société (traduit de l'allemand par J. Freund et al.), Paris, Plon (1 $1^{\text {ère }}$ éd. 1921), 411 p.

WEINSTOCK Daniel (2000) Démocratie et délibération, Archives de philosophie, 63, pp. 405-421. WILLIS Paul (1978) L'école des ouvriers, Actes de la recherche en sciences sociales, 24, pp. 51-61. ZINE Jasmin (2008) Canadian Islamic Schools, Toronto, Toronto University Press, 369 p. 


\title{
La négociation des frontières ethniques dans l'espace scolaire : un regard québécois
}

\author{
Stéphanie Tremblay
}

Cet article propose d'examiner, sous l'angle de la sociologie des relations ethniques et de la philosophie politique, les débats conceptuels et normatifs à propos de la construction des frontières ethniques dans l'espace scolaire. Dans un premier temps, l'auteure procède à une revue critique des principales approches théoriques de l'ethnicité et de leurs impacts sur les politiques scolaires. Puis, en souscrivant à une perspective libérale, qui articule le respect des droits individuels à une certaine reconnaissance des identités communautaires dans l'espace public, l'auteure tente dans un deuxième temps de délimiter la place légitime de la diversité culturelle et religieuse dans l'école libérale. En puisant notamment dans les débats québécois, qui touchent aussi à des degrés divers la plupart des sociétés pluralistes, l'auteure analyse donc les enjeux de la diversité au fil des trois grandes fonctions de la scolarisation : l'instruction, la socialisation et la qualification.

\section{The Negotiation of Ethnic Relations in School Space: A Quebec's Eye View}

\author{
Stéphanie Tremblay
}

The object of this article is to explore, through the lens of sociology of ethnic relations and political philosophy, the current conceptual and policy debates surrounding the construction of ethnic relations in the School space. In a first part, the author provides a critical review of the principal theorical approaches of ethnicity and their consequences on School policies. Furthermore, following a liberal perspective, which joins the respect of individual rights and a recognition of collective identities in public space, the author continues by trying to delimite the legitimate place of the cultural and religious diversity in the liberal School. Drawing example from debates in Quebec, which also concerns other pluralistic societies, the author analyses the diverse stakes of diversity through the three fonctions of liberal schooling : instruction, socialization and qualification.

\section{La negociación de fronteras étnicas en el espacio escolar: una mirada quebequesa}

\author{
Stéphanie Tremblay
}

Este artículo propone examinar, desde la perspectiva de la sociologia de las relaciones étnicas y de la filosofía política, los debates conceptuales y normativos sobre la construcción de fronteras étnicas en el espacio escolar. En un comienzo, la autora realiza una revision crítica de las principales perspectivas teóricas sobre la etnicidad y de su impacto sobre las políticas escolares. Luego, empleando una perspectiva liberal que articula el respeto de los derechos individuales a un recononocimeinto de las identidades comunitarias en el espacio público, la autora intenta delimitar el espacio legítimo de la diversidad cultural y religiosa en la escuela liberal. Tomando fundamentalmente los debates en Québec, que tocan igualmente en grados diversos la mayor parte de las sociedades pluralistas, la autora analiza la problemática de la diversidad a lo largo de tres grandes funciones de la escolarización : la instrucción, la socialización y la formación. 